\title{
An investigation in the estimation of body height using the length of ulna in healthy adults from Indian and Pakistani ethnic groups
}

\author{
M. Makda ${ }^{1}$, F. Amirabdollahian ${ }^{1}$ and A.M. Madden ${ }^{2}$ \\ ${ }^{1}$ School of Health Sciences, Liverpool Hope University, Liverpool, L16 9JD, UK and ${ }^{2}$ School of Life and Medical \\ Sciences, University of Hertfordshire, Hatfield, AL10 9AB, UK
}

The Malnutrition Universal Screening Tool (MUST) is developed to screen the risk of malnutrition among adults. Height measurements are needed for MUST and also in healthcare settings (e.g. for the purpose of the estimation of the energy requirements), but its measurement is a challenge for patients who cannot stand up. For such patients, a MUST equation can be used to predict height using ulna length ${ }^{1}$. However, the MUST equation does not take into consideration different ethnic backgrounds and the potential impact of ethnicity on the relationship between height and ulna length ${ }^{2}$. The aim of this study, therefore, is to investigate how accurately height can be estimated from ulna length in adults of Indian and Pakistani ethnicity using the MUST equation and to formulate more accurate ethnic specific equations, if necessary.

Ulna length and standing height was measured in 127 participants of Indian/Pakistani ethnicity, 67 males and 60 females. The participants were healthy volunteers aged 21-65 years. Height was predicted using the left ulna lengths firstly through the MUST equations and secondly compared against the measured values using an independent samples t-test. Regression formulas were then created for males and females.

The mean height (SD) was $171.0(6 \cdot 5) \mathrm{cm}$ and $155.7(5.8) \mathrm{cm}$ for men and women respectively. Ulna lengths and height were significantly correlated in both males $(r=0.645, p<0.01)$ and females $(r=0.596, p<0.01)$. There was a significant difference between the mean predicted height using MUST equation and mean measured height of participants for men $(177.2 \mathrm{~cm} \mathrm{vs.} 171.0 \mathrm{~cm}, \mathrm{p}<0.001)$ and women $(163.8 \mathrm{~cm}$ vs. $155.7 \mathrm{~cm}, \mathrm{p}<0.001)$ respectively. The current prediction equations of MUST show systematic overestimation of height in Indian and Pakistani men and women (Figure 1).
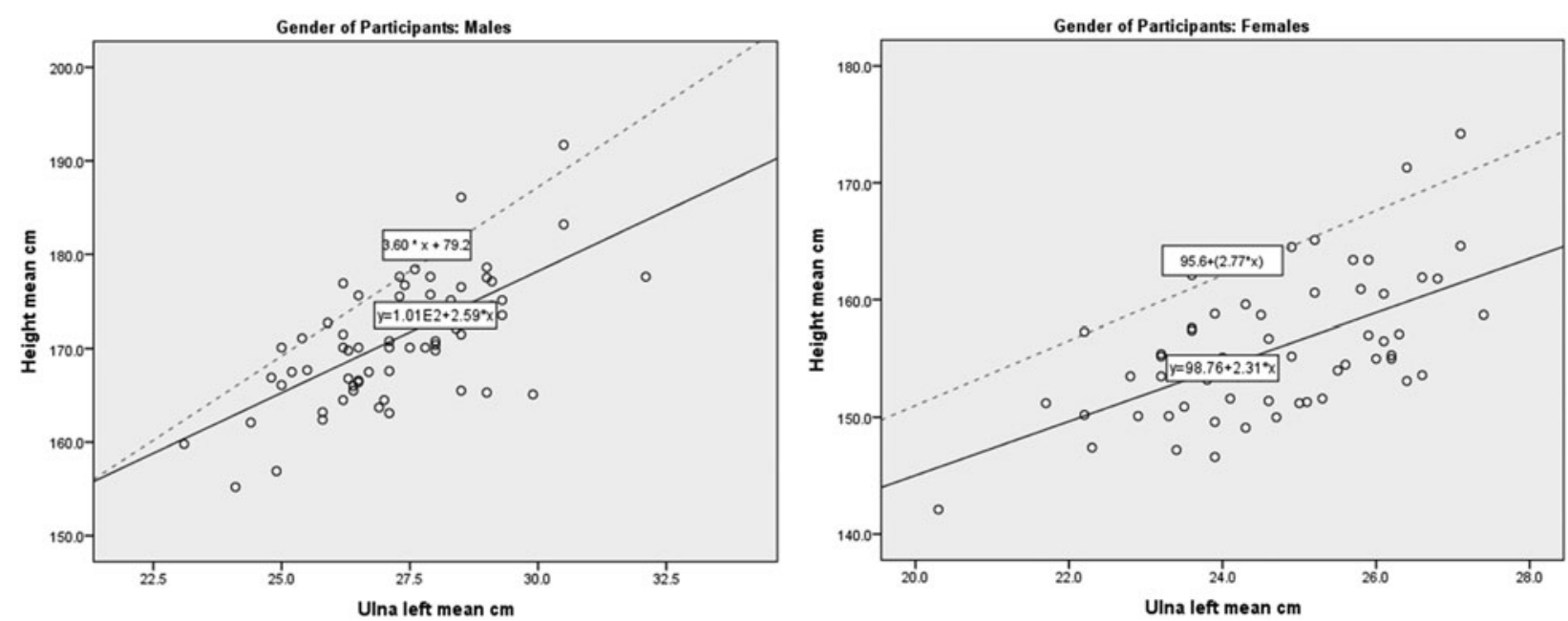

Fig. 1. Distribution of measured height against ulna length in males (left) and females (right). The dotted line represents the regression line for the appropriate MUST equation and the solid line represents the regression line for the values from the Indian and Pakistani participants.

The MUST equations for predicting height from ulna length in healthy adults should not be used among the Pakistani and Indian population as it overestimates the height, which could increase errors not only in the assessment of the risk of malnutrition but also in estimating energy requirement of patients. The results confirm previous findings ${ }^{2}$; however this will be soon followed by a larger study in the field. This is preliminary data of a multi-central collaborative project running in several research centres to produce prediction equation formulae for estimation of body height from the length of ulna within a representative sample of different ethnic groups across the UK.

1. Stratton RJ, King CL, Stroud MA et al. (2006) Br J Nutr 95, 325

2. Madden AM, Tsikoura T, Stott DJ. (2011) J Hum Nutr Diet 25, 21-128. 